\title{
The Incidence, Risk Factors, and Outcomes of Hyperlactatemia after Heart Transplantation One Center's Experience
}

\author{
Yasuhiro Hoshino, ${ }^{1} \mathrm{MD}$, Osamu Kinoshita, ${ }^{1} \mathrm{MD}$ and Minoru Ono, ${ }^{1} \mathrm{MD}$
}

\begin{abstract}
Summary
Hyperlactatemia (HL) is associated with tissue hypoperfusion during cardiac surgery, which results in postoperative morbidity and mortality among patients undergoing cardiopulmonary bypass surgery. The aim of this study was to determine the incidence, risk factors, and outcome of HL after heart transplantation (HTx) in one of the largest Japanese single-center cohorts. We retrospectively studied the lactate levels in 49 patients who underwent HTx at the University of Tokyo Hospital from August 1, 2010 to November 30, 2015. All of the patients were over 20 years of age. Arterial blood samples were analyzed during the operation and until 24 hours after surgery. Twenty-nine patients (59.2\%) had HL after intensive-care unit admission. At 24 hours after surgery, the lactate levels of all patients had recovered to the normal range. A multivariate analysis showed that the total ischemic time of the donor heart (odds ratio [OR], 1.0176; 95\% confidence interval [CI], 1.0004-1.0375; $P$ $=0.0444)$ and the duration of preoperative left ventricular assist device (LVAD) support (OR, 0.9977; 95\% CI, $0.9952-0.9997 ; P=0.0218$ ) were risk factors for HL. Pulmonary complications were noted in $24.1 \%$ of the patients with high lactate values but in none of the patients without HL $(P=0.0182)$; however, there were no cases of hospital death, and the length of hospital stay did not differ to a statistically significant degree between HL groups $(P=0.719)$. Although HL after HTx was common, it appeared to be transient and benign. Donor heart ischemia and the duration of preoperative LVAD support were associated with HL after transplantation.
\end{abstract}

Key words: Donor heart ischemia

(Int Heart J 2018; 59: 81-86)

$\mathrm{L}$ actic acidosis is related to tissue hypoperfusion and hypoxia in critically ill patients with sepsis ${ }^{1)}$ with a poor prognosis when left untreated. Under anaerobic conditions, cellular metabolism converts pyruvate to lactate. The lactic acid levels, which are related to the oxygen debt, correspond to the severity of tissue hypoperfusion, and lactic acidosis or hyperlactatemia (HL) is a well-recognized marker of circulatory failure.

$\mathrm{HL}$ is a common phenomenon, especially during and after cardiac surgery with cardiopulmonary bypass $(\mathrm{CPB}),{ }^{5)}$ and is associated with postoperative morbidity and mortality. ${ }^{6}$ Several mechanisms have been proposed to explain its physiopathogenesis. Some reports suggest that the degree of hemodilution and low peripheral oxygen delivery leads to tissue hypoxia. ${ }^{7.8}$ Similarly, splanchnic and renal hypoperfusion are likely to lead to a reduced lactate clearance. A relationship has been suggested between lactic acidosis and the reduction of the preoperative afterload by angiotensin-converting enzyme inhibitors (ACEIs) or other drugs that are administered preoperatively to treat heart failure.")

No reports have so far described the incidence, risk factors, or outcomes of HL after HTx in Japan. The aims of the present study were to assess the prognostic significance of $\mathrm{HL}$ and to determine the incidence and risk factors for HL after HTx in one of the largest Japanese single-center cohorts.

\section{Methods}

Study patients: The study included 54 consecutive patients who had undergone HTx at the University of Tokyo Hospital between August 2011 and November 2015. After excluding five patients who were younger than 20 years of age, a total of 49 patients were included in the analysis. This study complied with the Declaration of Helsinki, and written informed consent was obtained from each patient regarding the use of his or her clinical data.

The patients were divided into two groups based on the peak lactate level within 24 hours after HTx. The HL group included patients whose peak lactate level was $\geq 4$ $\mathrm{mmol} / \mathrm{L}$; all other patients were classified into the nonhyperlactatemia (NHL) group. We set $4 \mathrm{mmol} / \mathrm{L}$ as the cutoff level based on the threshold of early goal-directed therapy in the treatment of severe sepsis and septic

From the ${ }^{1}$ Department of Cardiac Surgery, The University of Tokyo Hospital, Tokyo, Japan.

Address for correspondence: Yasuhiro Hoshino, MD, Department of Cardiac Surgery, The University of Tokyo Hospital, 7-3-1 Hongo, Bunkyo-Ku, Tokyo 113-8655, Japan. E-mail: pwd8knd273@yahoo.co.jp

Received for publication March 15, 2017. Revised and accepted May 3, 2017

Released in advance online on J-STAGE December 27, 2017.

doi: 10.1536/ihj.17-146

All rights reserved by the International Heart Journal Association. 
shock. ${ }^{10)}$

HTx procedures: All donor hearts were examined by echocardiography and explanted from suitable donors. During organ procurement, the donor hearts were arrested and preserved with Celsior solution (Pasteur Merieux, Paris, France). The total ischemic time was $254.3 \pm 38.3$ minutes. The anesthesia techniques and medications were similar for all patients. Anesthesia was induced and maintained using a combination of propofol, fentanyl, or midazolam and a neuromuscular paralytic agent. Most patients received an inhaled anesthetic (sevoflurane). Standard median sternotomy and aorta/bicaval cannulation were performed for CPB. The pump circuit was primed with lactate Ringer solution (L-lactate: 11.2-19.6 mEq). The rectal temperature was cooled to $28^{\circ} \mathrm{C}$. The nonpulsatile blood flow was adjusted to maintain a blood flow of approximately 2.6-2.7 $1 /$ minute $/ \mathrm{m}^{2}$. Red blood cell transfusion was performed as necessary to maintain a hematocrit level of approximately $23 \%-24 \%$. All patients were retransfused with lost blood (Cell Saver; Haemonetics Corporation, Braintree, MA, USA). Vaporized isoflurane, nitroglycerin, phenylephrine hydrochloride, or norepinephrine was continuously infused to maintain a perfusion pressure of approximately $50-70 \mathrm{mmHg}$. Following standard rewarming and de-airing, the pump was discontinued with routine inotropic support, including dopamine at $2-8 \mu \mathrm{g} / \mathrm{kg} /$ minute and low-dose phosphodiesterase III inhibitor treatment to obtain an acceptable cardiac output and isoproterenol or temporary pacing to achieve a heart rate of approximately 90-100 beats/minute. If necessary, dobutamine, epinephrine, and/or norepinephrine infusion was started. In patients with an unstable cardiopulmonary condition, an intra-aortic balloon pump (IABP, $n=4$ ) was used or extracorporeal membrane oxygenation (ECMO, $n=2)$ was performed. All patients were sent to the intensive-care unit (ICU) postoperatively with endotracheal intubation. Our standard immunosuppressive regimen includes calcineurin inhibitor, mycophenolate mofetil, and high-dose methylprednisolone.

The lactate level was measured when entering the ICU and thereafter every 6 hours until 24 hours after the operation, and it was additionally measured according to the ICU physician's policy using an ABL 800 FLEX device (RADIOMETER; Copenhagen, Denmark). The lactate (detectable limit, $0.0-30 \mathrm{mmol} / \mathrm{L}$ ) and glucose (detectable limit, 0-1,081 mg/dl) levels were measured.

Major neurological complications were defined as seizure, stroke, severe diffuse encephalopathy, or severe delirium. Pulmonary complications were defined as mechanical ventilation for $>72$ hours. Renal complications were defined as elevated postoperative creatinine levels of $>2.5 \mathrm{mg} / \mathrm{dL}$ or the need for hemodialysis or hemofiltration. Graft dysfunction on the first echocardiogram after transplantation was defined as an ejection fraction $<45 \%$. Mortality was defined as hospital mortality.

Statistical analyses: All of the data were expressed as the mean \pm standard deviation or as a percentage. The differences between the two groups were assessed with either Fisher's exact test or a $t$-test, as appropriate. Two-sided $P$ values of $<0.05$ were considered to indicate statistical significance. A univariate analysis on the risk of HL after
HTx was performed on 43 factors before, during, and after surgery. With respect to factors whose $P$ value was < 0.05 among univariate analyzed 43 factors, correlation coefficients and their $P$ values were calculated for all combinations in consideration of multicollinearity among factors. When correlation was observed, one factor was excluded from factors of multivariate analysis. Finally, the factors with no correlation were adopted for a multivariate logistic regression model. Odds ratios (ORs) and 95\% confidence intervals (CIs) were calculated. The statistical analyses were performed using the $\mathrm{JMP}^{\circledR}$ Pro software program (version 11.2.0; SAS Institute; Cary, NC, USA).

\section{Results}

The patients' characteristics are shown in Table I. HL was observed in 29 of the $49(59.2 \%)$ patients. The mean lactate peak value was $5.9 \pm 3.5 \mathrm{mmol} / \mathrm{L}$, and the time to peak lactate was $3.1 \pm 3.7$ hours. The average age was $42.4 \pm 11.6$ years, $71.4 \%$ of patients were men, and the etiology of heart failure was dilated cardiomyopathy in $65.3 \%$ of the patients. With the exception of the duration of preoperative left ventricular assist device (LVAD) support, the two groups had similar preoperative characteristics. The duration of LVAD support was shorter among the patients in the postoperative HL group than in the NHL group $(791.0 \pm 315.0$ versus $1038.8 \pm 360.0, P=$ $0.0149)$. None of the patients used insulin before surgery. None of the patients had renal or liver dysfunction. The use of ACEIs did not differ markedly between the two groups. The preoperative LVAD flow conditions (continuous or pulsatile) did not differ significantly between the two groups.

The operative data are shown in Table II. The duration of surgery, duration of CPB, amount of blood loss and blood transfusion, maximum dose of catecholamine, and the mean CPB flow did not differ markedly between the two groups. The total ischemic time of the donor heart in the HL group was longer than that in the NHL group (264.6 \pm 36.2 versus $239.4 \pm 37.1, P=0.0218)$.

The postoperative data are shown in Table III. Cardiopulmonary instability, including the need for mechanical support, did not differ markedly between the two groups. The postoperative peak blood glucose and the glucose at the time of peak lactate level (GPL) were significantly higher in the HL group than in the NHL group $(P$ $=0.0425, P=0.0053)$. There were no significant differences between the two groups with regard to the amount of insulin used during the first 24 hours in the ICU.

A univariate analysis of the risk of HL after HTx was performed on 43 factors before, during, and after surgery, and three factors had $P$ values $<0.05$ (postoperative GPL, duration of preoperative LVAD support, and total ischemic time of the donor heart; $P=0.0030,0.0119$, 0.0193 , respectively). The correlation coefficients and the levels of significance were calculated for all combinations (Table IV). A correlation was found between the postoperative GPL and the total ischemic time of the donor heart. Because the total ischemic time of the donor heart is fixed during a procedure and the postoperative GPL is measured postoperatively, the temporal context was clear 
Table I. The Preoperative Characteristics

\begin{tabular}{lccc}
\hline \multicolumn{1}{c}{ Variable } & NHL $(n=20)$ & HL $(n=29)$ & $P$ \\
\hline Age, years & $42.6 \pm 2.6$ & $42.3 \pm 2.2$ & 0.9245 \\
Male gender, $n(\%)$ & $13(65)$ & $22(75.9)$ & 0.5237 \\
Body mass index & $21.1 \pm 2.9$ & $20.4 \pm 3.5$ & 0.4533 \\
Left ventricular ejection fraction, $\%$ & $28.0 \pm 18.2$ & $23.0 \pm 13.8$ & 0.2852 \\
Pre-transplant etiology & & & \\
Idiopathic dilated CM $(n=32), n(\%)$ & $12(60)$ & $20(69.0)$ & 0.5548 \\
Ischemic CM $(n=7), n(\%)$ & $6(30)$ & $1(3.4)$ & \\
Dilated hypertrophic CM $(n=6), n(\%)$ & $1(5)$ & $5(17.2)$ & \\
Other $(n=4)$ & & & \\
Pretransplant LVAD support duration, days $(n=48)$ & $1038.8 \pm 360.0$ & $791.0 \pm 315.2$ & 0.0149 \\
CF-LVAD, $n(\%)$ & $10(50)$ & $21(72.4)$ & 0.1388 \\
Hct, $\%$ & $34.8 \pm 6.7$ & $36.4 \pm 6.6$ & 0.4116 \\
Total bilirubin, mg/dL & $1.0 \pm 0.4$ & $0.9 \pm 0.4$ & 0.4047 \\
AST, IU/mL & $31.6 \pm 14.3$ & $35.0 \pm 18.3$ & 0.4938 \\
ALT, IU/mL & $16.3 \pm 7.8$ & $22.8 \pm 20.1$ & 0.1770 \\
Creatinin, mg/dL & $1.0 \pm 0.3$ & $1.0 \pm 0.3$ & 0.9993 \\
ACEI use, $n(\%)$ & $10(50)$ & $14(48.3)$ & 1.0 \\
\hline LVAD indictes left & &
\end{tabular}

LVAD indicates left ventricular assist device support; CF-LVAD, continuous flow LVAD; AST, aspartate aminotransferase; ALT, alanine aminotransferase; Hct, hematocrit; ACEI, angiotensin-converting enzyme inhibitor; and CM, cardiomyopathy.

Table II. The Operative Data

\begin{tabular}{|c|c|c|c|}
\hline Variable & NHL $(n=20)$ & $\mathrm{HL}(n=29)$ & $P$ \\
\hline \multicolumn{4}{|l|}{ Donor heart characteristics } \\
\hline Total ischemic time of the donor heart, minutes & $239.4 \pm 37.1$ & $264.6 \pm 36.2$ & 0.0218 \\
\hline Age, years & $45.8 \pm 13.8$ & $45.4 \pm 12.1$ & 0.9180 \\
\hline Male gender, $n(\%)$ & $11(55)$ & $15(52)$ & 1.0000 \\
\hline LVEF, \% & $64.5 \pm 8.6$ & $64.2 \pm 7.3$ & 0.9045 \\
\hline History of CPR, $n(\%)$ & $7(35)$ & $15(52)$ & 0.3812 \\
\hline Dopamine dose, $\mu \mathrm{g} / \mathrm{kg} / \mathrm{minute}$ & $4.9 \pm 6.0$ & $4.4 \pm 4.2$ & 0.7738 \\
\hline Noradrenaline dose, $\mu \mathrm{g} / \mathrm{kg} /$ minute & $0.05 \pm 0.2$ & $0.02 \pm 0.05$ & 0.3216 \\
\hline Cause of brain death (cerebrovascular accident or not), $n(\%)$ & $14(70)$ & $23(79)$ & 0.5123 \\
\hline Surgery duration, minutes & $586.1 \pm 23.5$ & $609.9 \pm 19.5$ & 0.4397 \\
\hline $\mathrm{CPB}$ duration, minutes & $179.1 \pm 41.2$ & $193.8 \pm 59.5$ & 0.3435 \\
\hline Blood loss, $\mathrm{mL}$ & $4663.8 \pm 2000.5$ & $4400.7 \pm 2964.8$ & 0.7311 \\
\hline Dopamine, $\mu \mathrm{g} / \mathrm{kg} /$ minute & $6.8 \pm 2.0$ & $6.3 \pm 2.1$ & 0.3850 \\
\hline Dobutamine, $\mu \mathrm{g} / \mathrm{kg} /$ minute & $6.9 \pm 2.0$ & $6.1 \pm 2.1$ & 0.1821 \\
\hline Epinephrine, $\mu \mathrm{g} / \mathrm{kg} /$ minute & $0.073 \pm 0.15$ & $0.057 \pm 0.13$ & 0.6910 \\
\hline Norepinephrine, $\mu \mathrm{g} / \mathrm{kg} /$ minute & $0.06 \pm 0.06$ & $0.14 \pm 0.28$ & 0.2329 \\
\hline Red blood cell, units & $16.5 \pm 7.2$ & $17.9 \pm 10.9$ & 0.6054 \\
\hline Fresh-frozen plasma, units & $17.9 \pm 7.4$ & $20.8 \pm 12.3$ & 0.3596 \\
\hline Platelet concentrate, units & $34.5 \pm 14.3$ & $33.8 \pm 13.7$ & 0.8626 \\
\hline Peak blood glucose, $\mathrm{mg} / \mathrm{dL}$ & $222.2 \pm 36.2$ & $249.1 \pm 62.1$ & 0.0882 \\
\hline Minimum Hct, \% & $22.0 \pm 3.5$ & $21.7 \pm 2.9$ & 0.6910 \\
\hline Mean CPB flow, $\mathrm{mL} /$ minute & $3746.5 \pm 461.4$ & $3798.2 \pm 576.2$ & 0.7400 \\
\hline
\end{tabular}

CPB indicates cardiopulmonary bypass; LVEF, left ventricular ejection fraction; and CPR, cardiopulmonary resuscitation.

for the postoperative GPL and the total ischemic time of the donor heart, so only the postoperative GPL was excluded as a factor from the multivariate analysis. We ultimately subjected two factors (total ischemic time of the donor heart and duration of preoperative LVAD support) to our multivariate analysis. Both the total ischemic time of the donor heart (OR, 1.0176; 95\% CI, 1.0004-1.0375; $P=0.0444)$ and the duration of preoperative LVAD support (OR, 0.9977; 95\% CI, 0.9952-0.9997; $P=0.0218$ ) were found to be independent risk factors for $\mathrm{HL}$ after HTx.
The post-transplant outcomes are shown in Table V. Postoperative pulmonary complications occurred more frequently in the HL group than in the NHL group $(24.1 \%$ versus $0 \%, P=0.0322$ ) due to the longer duration of intubation in the HL group $(46.2 \pm 59.2$ versus $15.2 \pm 11.2$, $P=0.025)$. There were no significant differences between the two groups with regard to graft dysfunction. No hospital deaths occurred in either group. 
Table III. The Postoperative Data

\begin{tabular}{lccl}
\hline \multicolumn{1}{c}{ Variable } & NHL $(n=20)$ & HL $(n=29)$ & $P$ \\
\hline Peak blood glucose, $\mathrm{mg} / \mathrm{dL}$ & $215 \pm 38.9$ & $242.2 \pm 48.6$ & 0.0425 \\
GPL, $\mathrm{mg} / \mathrm{dL}$ & $176.0 \pm 42.6$ & $222.0 \pm 60.8$ & 0.0053 \\
Dopamine, $\mu \mathrm{g} / \mathrm{kg} /$ minute & $4.7 \pm 3.0$ & $4.8 \pm 2.1$ & 0.9169 \\
Dobutamine, $\mu \mathrm{g} / \mathrm{kg} /$ minute & $5.4 \pm 2.2$ & $5.1 \pm 2.3$ & 0.6816 \\
Epinephrine, $\mu \mathrm{g} / \mathrm{kg} /$ minute & $0.08 \pm 0.25$ & $0.11 \pm 0.33$ & 0.7511 \\
Norepinephrine, $\mu \mathrm{g} / \mathrm{kg} /$ minute & $0.6 \pm 1.2$ & $0.7 \pm 1.4$ & 0.8228 \\
Epinephrine use, $n(\%)$ & $2(10.0)$ & $3(10.3)$ & 1.0 \\
Mechanical support, $n(\%)$ & $1(5.0)$ & $5(17.2)$ & 0.3794 \\
Amount of insulin*, $\mathrm{U}$ & $25.4 \pm 24.6$ & $40.5 \pm 36.5$ & 0.1138 \\
\hline
\end{tabular}

GPL indicates glucose at the time of peak lactate level: and*, Amount of insulin during the first 24 hours in the intensive-care unit.

Table IV. Correlation Matrix with Significance Levels ( $P$ Value) for Factors Significantly Associated with HL after Surgery on Univariate Analysis

\begin{tabular}{lccc}
\hline \multicolumn{1}{c}{ Factor } & $\begin{array}{c}\text { Postoperative glucose } \\
\text { at peak lactate levels }\end{array}$ & $\begin{array}{c}\text { Duration of preoperative } \\
\text { LVAD support }\end{array}$ & $\begin{array}{c}\text { Total ischemic time } \\
\text { of the donor heart }\end{array}$ \\
\hline Postoperative glucose at peak lactate levels & $1.0000(<0.0001)$ & $-0.1214(0.4113)$ & $0.3070(0.0319)$ \\
Duration of preoperative LVAD support & $-0.1214(0.4113)$ & $1.0000(<0.0001)$ & $-0.1484(0.3143)$ \\
Total ischemic time of the donor heart & $0.3070(0.0319)$ & $-0.1484(0.3143)$ & $1.0000(<0.0001)$ \\
\hline
\end{tabular}

HL indicates hyperlactatemia; and LVAD, left ventricular assist device support.

Table V. The Outcomes

\begin{tabular}{lccc}
\hline \multicolumn{1}{c}{ Variable } & NHL $(n=20)$ & HL $(n=29)$ & $P$ \\
\hline Pulmonary complication, $n(\%)$ & $0(0)$ & $7(24.1)$ & 0.0322 \\
Renal complication, $n(\%)$ & $4(20)$ & $8(27.6)$ & 0.7377 \\
Neurological complication, $n(\%)$ & $2(10.0)$ & $3(10.3)$ & 1.0000 \\
Graft dysfunction, $n(\%)$ & $0(0)$ & $2(6.9)$ & 0.5068 \\
Hospital stay, days & $79.8 \pm 130.7$ & $61.4 \pm 40.8$ & 0.4808 \\
Hospital mortality, $n(\%)$ & $0(0)$ & $0(0)$ & \\
\hline
\end{tabular}

$\mathrm{CPB}$ indicates cardiopulmonary bypass; and Hct, hematocrit.

\section{Discussion}

The main findings of the present study were as follows: (1) HL occurred after HTx in $59.2 \%$ of this population, (2) HL appears to be independently associated with the total ischemic time of the donor heart and the duration of preoperative LVAD support, and (3) HL is not associated with unfavorable outcomes other than pulmonary complications.

The incidence of HL in our study was almost the same as that of previous studies $;{ }^{11}$ however, it was thought to be higher than the incidence reported in previous studies on general heart surgery. ${ }^{6,12)} \mathrm{Hsu}$, et al. ${ }^{13)} \mathrm{re}-$ ported that HL occurred in 12 of 58 patients $(21 \%)$. As the cutoff level of HL was $15 \mathrm{mmol} / \mathrm{L}$ in that study, the incidence in their population might have been higher given the difference in the cutoff level. We also considered the possibility that their population might have included patients in an unstable cardiopulmonary state prior to surgery (preoperative use of IABP, $n=5$; ECMO, $n=$ 2, maximum preoperative lactate level, $3.1 \mathrm{mmol} / \mathrm{L}$ ), as their report included four cases of hospital mortality (4 of $12,33 \%)$.

According to previous reports, the risk factors for $\mathrm{HL}$ in both HTx and general cardiac surgery are related to the duration of $\mathrm{CPB}$, the duration of surgery, the intraoperative use of vasopressors, the maximum dose of inotropic drugs during the reperfusion phase and 12-24 hours after surgery, and the duration of inotrope use..$^{6,11,14)}$ In the present study, we found no significant difference between the groups with regard to the maximum dose of inotropic drugs during the reperfusion phase and at 12-24 hours after surgery, the duration of CPB, the duration of surgery, or the duration of inotrope use. However, these factors were not significant in our study because the heart function is maintained in patients after HTx, and in many cases, the circulation dynamics are stable. Nixon, et al. reported on the association between preoperative mechanical support and HL. ${ }^{11)}$ They found that HL was caused by a longer ischemic time due to complicated redo surgery, such as cases in which an LVAD was used. In our study, except for one case, an LVAD was implanted in all cases prior to HTx. The multivariate analysis revealed that both the total ischemic time of the donor heart and the duration of pretransplant LVAD were independent risk factors for HL after HTx. These findings suggest that HL may be caused by lactate flowing out of the graft. During cardiac surgery, lactate is measured from the coronary sinus after reperfusion of the heart over time, Chowdhury, et al. reported that lactate increased as myocardial damage 
strengthened. ${ }^{15)}$ This suggests that lactate accumulates in the heart as a result of the slow progression of myocardial injury, despite being stored in cooled cardiac preservation solution. Lactate then flows throughout the body after reperfusion, and HL is observed in arterial blood. The results of this study suggest that with this degree of HL, metabolic disorders leading to cardiac dysfunction and multiple organ disorder can be avoided.

We consider the association between $\mathrm{HL}$ and the shorter duration of the LVAD support to be due to instability of the heart failure condition before transplantation. First, as reported by Mohacsi, et al., exercise tolerance declines in patients with heart failure and tends to produce lactic acid. ${ }^{14)}$ Heart failure patients, even those with a preserved cardiac output (cardiac index $>21 /$ minute $/ \mathrm{m}^{2}$ ), can have a severe reduction in their peak oxygen consumption in preoperative spiroergometry. Poor exercise tolerance is due to early anaerobic metabolism. These patients are expected to have similar metabolic tendencies after transplantation and are likely to develop HL after transplantation. Regarding the characteristics of the donor hearts, although left ventricular hypertrophy (left ventricular wall thickness) and the body weight ratio were not considered, differences between the groups were observed in the total ischemic time of the donor heart, and there is a possibility that marginal donor hearts were used more frequently in the HL group (Table II). In other words, patients whose heart failure status before transplantation was unstable, may have been performed HTx earlier using marginal donor hearts, and as a result, the possibility that pretransplant LVAD support became shorter was considered. However, regarding patient characteristics, no right-sided heart catheters were inserted nor was spiroergometry performed immediately before transplantation, and further examinations of exercise tolerance are needed before surgery.

The results of this study indicate that the duration of intubation was significantly longer in the HL group than in the NHL group, and pulmonary complications were more frequent. However, HL is transient after surgery and recovered to the normal range within 24 hours. As such, a direct effect of HL on pulmonary complications is unlikely. Therefore, we speculate that short-term LVAD support, which is the determinant factor of HL, is associated with pulmonary complications. The mechanism underlying this relationship is thought to be the existence of heart failure before transplantation. Kinugawa, et al. reported that exercise intolerance related to skeletal muscle adversely affects not only prognosis but also quality of life in patients with heart failure. ${ }^{16)}$ There is also a report such as respiratory dysfunction after transplantation due to deconditioning of the respiratory muscles before transplantation. ${ }^{17)}$ Moreover, Mayer, et al. reported that patients with heart failure had a decreased respiratory muscle function, which was associated with their prognosis. ${ }^{18)}$ We therefore hypothesize that patients with heart failure who have low exercise tolerance because of a low respiratory muscle function tend to develop postoperative $\mathrm{HL}$ and may be prone to pulmonary complications.

We were unable to assess the involvement of systemic tissue hypoxia, as we did not examine the intracellular NAD+/NADH ratio. Further studies are therefore needed to determine the metabolic state of systemic tissue using microdialysis in HTx patients.

Clinical implications: $\mathrm{HL}$ is associated with the total ischemic time of the donor heart and the duration of preoperative LVAD support. The former is one of the indicators that characterize the donor heart, and the latter is considered to be one of the indicators of preoperative heart failure status. HL is a good marker of the total ischemic time of the donor heart and the duration of preoperative LVAD support, and it can predict pulmonary complications after transplantation.

Study limitations: The present study is associated with two limitations: the study was retrospective in nature and was based on a single hospital experience; thus, these findings may not be generalizable.

\section{Conclusion}

We found that HL was common after HTx. The total ischemic time of the donor heart and the duration of preoperative LVAD support were risk factors for HL after HTx. Further prospective studies are needed to confirm the association between HL and both of donor heart ischemia and heart failure status before transplantation.

\section{Disclosures}

Conflicts of interest: The authors declare no conflicts of interest in association with the present study.

\section{References}

1. Mizock BA, Falk JL. Lactic acidosis in critical illness. Crit Care Med 1992; 20: 80-93.

2. Broder G, Weil MH. Excess lactate: an index of reversibility of shock in human patients. Science 1964; 143: 1457-9.

3. Weil MH, Afifi AA. Experimental and clinical studies on lactate and pyruvate as indicators of the severity of acute circulatory failure (shock). Circulation 1970; 41: 989-1001.

4. Vitek V, Cowley RA. Blood lactate in the prognosis of various forms of shock. Ann Surg 1971; 173: 308-13.

5. Mak NT, Iqbal S, de Varennes B, Khwaja K. Outcomes of postcardiac surgery patients with persistent hyperlactatemia in the intensive care unit: a matched cohort study. J Cardiothorac Surg 2016; 11: 33 .

6. Maillet JM, Le Besnerais P, Cantoni M, et al. Frequency, risk factors, and outcome of hyperlactatemia after cardiac surgery. Chest 2003; 123: 1361-6.

7. Raper RF, Cameron G, Walker D, Bowey CJ. Type B lactic acidosis following cardiopulmonary bypass. Crit Care Med 1997; 25: 46-51.

8. Habib RH, Zacharias A, Schwann TA, Riordan CJ, Durham SJ, Shah A. Adverse effects of low hematocrit during cardiopulmonary bypass in the adult: should current practice be changed? J Thorac Cardiovasc Surg 2003; 125: 1438-50.

9. Robbins RC, Smith JA, Rihakove GH, et al. Low systemic resistance syndrome following heart transplantation. In: 14th Annual Meeting of the American Society of Transplant Physicians. Chicago: Abstract booklet (P-72); 1994: 143.

10. Rivers E, Nguyen B, Havstad S, et al. Early goal-directed therapy in the treatment of severe sepsis and septic shock. N Engl J Med 2001; 345: 1368-77.

11. Nixon JL, Kfoury AG, McCubrey R, et al. Lactic acidosis after cardiac transplantation: foe or common innocent bystander? Transplantation 2015; 99: 1216-9. 
12. Demers P, Elkouri S, Martineau R, Couturier A, Cartier R. Outcome with high blood lactate levels during cardiopulmonary bypass in adult cardiac operation. Ann Thorac Surg 2000; 70: 2082-6.

13. Hsu YC, Hsu CH, Huang GS, et al. Extreme hyperlactatemia after heart transplantation: One center's experience. Transplan Proc 2015; 47: 1945-8.

14. Mohacsi P, Pedrazzinia G, Tanner H, Tschanz HU, Hullin R, Carrel T. Lactic acidosis following heart transplantation: a common phenomenon? Eur J Heart Fail 2002; 4: 175-9.

15. Chowdhury UK, Sheil A, Kapoor PM, et al. Short-term prognostic value of perioperative coronary sinus-derived-serum cardiac troponin-I, creatine kinase-MB, lactate, pyruvate, and lactate-pyruvate ratio in adult patients undergoing open heart surgery. Ann Card Anaesth 2016; 19: 439-53.

16. Kinugawa S, Takada S, Matsushima S, Okita K, Tsutsui H. Skeletal muscle abnormalities in heart failure. Int Heart J 2015; 56: 475-84.

17. Coronel CC, Bordignon S, Bueno AD, Lima LL, Nesralla I. Perioperative variables of ventilatory function and physical capacity in heart transplant patients. Rev Bras Cir Cardiovasc 2010; 25: 190-6.

18. Meyer FJ, Borst MM, Zugck C, et al. Respiratory muscle dysfunction in congestive heart failure: clinical correlation and prognostic significance. Circulation 2001; 103: 2153-8. 